\title{
Comparison of Reusable Models in Pericardiocentesis Simulation Training
}

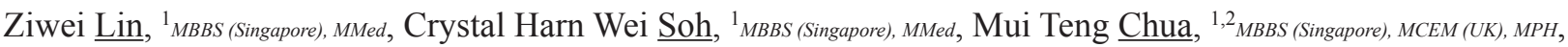
Jingping Lin, ${ }^{1}{ }_{M B B S}(L o n d o n), M M e d$, Cheryl Jing Yi Ho,${ }_{B A(I D)}$, Julia Ying Hui Lee, ${ }^{3}{ }_{B A}(I D)$, Fang Yu Tracy $\underline{\text { Shen, }},{ }_{B A}{ }_{B A}(I D)$, Ying Wei Yau, ${ }^{1,2}$ MBBS (Singapore), MMed, MCI, Win Sen Kuan, ${ }^{1,2}$ MBBS (Singapore), MRCSEd (A\&E), MCI

\begin{abstract}
Introduction: Pericardiocentesis is a potentially life-saving procedure. We compared two low-cost models - an agar-based model and a novel model, Centesys - in terms of ultrasound image quality and realism, effectiveness of the model, and learners' confidence and satisfaction after training.

Methods: In this pilot randomised $2 \times 2$ crossover trial stratified by physician seniority, participants were assigned to undergo pericardiocentesis training either with the agar-based or Centesys model first, followed by the other model. Participants were asked to rate their confidence in performing ultrasound-guided pericardiocentesis, clarity and realism of cardiac structures on ultrasound imaging, and satisfaction on a 7-point Likert scale before and after training with each model.

Results: Twenty participants with median postgraduate year of 4 (interquartile range [IQR] 3.75-6) years were recruited. Pre-training, participants rated themselves a median score of 2.5 (IQR 2-4) for level of confidence in performing pericardiocentesis, which improved to 5 (IQR 4-6) post-training with Centesys $(P=0.007)$. Centesys was recognised to be more realistic in simulating cardiac anatomy on ultrasound (median 5 [IQR 4-5] versus 3.5 [IQR 3-4], $P=0.002$ ) than the agar-based model. There was greater satisfaction with Centesys (median 5 [IQR 5-6] versus 4 [IQR 3.75-4], $P<0.001)$. All 20 participants achieved successful insertion of a pericardial drain into the simulated pericardial sac with Centesys.

Conclusion: Centesys achieved greater learner satisfaction as compared to the agar-based model, and was an effective tool for teaching ultrasound-guided pericardiocentesis and drain insertion.
\end{abstract}

Ann Acad Med Singap 2020;49:971-77

Keywords: Cardiac tamponade, emergency medicine, medical education, pericardial effusion, ultrasonography

\section{Introduction}

Pericardiocentesis is a potentially life-saving procedure for acute cardiac tamponade. ${ }^{1}$ However, this condition occurs rarely in clinical practice. ${ }^{2}$ Even with an experienced operator, pericardiocentesis is associated with dangerous complications such as cardiac perforation and pneumothorax. ${ }^{3}$ Even with echocardiogram-guided pericardiocentesis, the complication rate ranges from $3.7 \%$ to $10 \%{ }^{4-7}$ Simulation training provides regular hands-on practice to maintain competency in managing such an infrequently encountered condition. ${ }^{8}$

Ultrasound has been shown to be a useful modality in the emergency department, even in critical situations such as cardiac arrest. ${ }^{9}$ Ultrasound-guided pericardiocentesis is considered the standard of care, and has been associated with fewer complications and higher success rates compared to the traditional blind approach. ${ }^{5-7}$ Simulation models should hence

\footnotetext{
${ }^{1}$ Emergency Medicine Department, National University Hospital, National University Health System, Singapore

${ }^{2}$ Department of Surgery, Yong Loo Lin School of Medicine, National University of Singapore, Singapore

${ }^{3}$ Division of Industrial Design, School of Design \& Environment, National University of Singapore, Singapore

Address for Correspondence: Dr Ziwei Lin, National University Hospital, Emergency Medicine Department, Level 4, National University Centre for Oral Health, 9 Lower Kent Ridge Road, Singapore 119085.

E-mail: ziwei_lin@nuhs.edu.sg
} 
be made ultrasound-compatible for more realistic learning. Commercial models are costly, ranging from approximately US $\$ 3,000$ to $18,000 .{ }^{10,11}$ Do-it-yourself models have been described previously, using low-cost materials such as gel wax ${ }^{12,13}$ and tofu. ${ }^{2}$ However, these models did not accommodate insertion of larger-bore (6 to 8 French) pericardial drains and are not practical for long-term training as the materials disintegrate with repeated use. Cadaveric models have also been described, ${ }^{14}$ and while they may retain anatomical accuracy, these models are very costly and not readily available. ${ }^{15}$ For training purposes, our centre adopted an agar-based model, using agar jelly as the ultrasound medium to simulate subcutaneous tissues, constructed using the methodology previously described by Zerth et al. ${ }^{16}$

Recently, we constructed a novel model with focus on durability and improved fidelity to reduce the cost required for preparation and enhance training quality. This new model, named Centesys, was designed in collaboration with undergraduate students from the industrial design programme at the School of Design and Environment, National University of Singapore. The objectives of our study were to ascertain and compare the learners' confidence at performing ultrasound-guided pericardiocentesis before and after training with Centesys versus the agar-based model, the ultrasound image quality of both models at simulating cardiac anatomy, the learners' satisfaction with both models, and the effectiveness of Centesys and the agar-based model as training tools for ultrasoundguided pericardiocentesis.

\section{Methods}

We designed a pilot randomised $2 \times 2$ crossover trial, stratified by seniority (residents, senior residents and resident physicians). This stratification was to ensure groups would have similar proportions of senior and junior physicians in each group to minimise risk of prognostic imbalance from differential years of postgraduate experience. Ethics approval was obtained from the National Healthcare Group Domain Specific Review Board for exemption of written consent (DSRB reference number 2018/00543).

Participants were emergency medicine residents and resident physicians from the Emergency Medicine Department of National University Hospital, Singapore, a tertiary academic medical institution. We have an established 5-year emergency medicine residency programme accredited by Accreditation Council for
Graduate Medical Education International. ${ }^{17}$ Data was collected during a 45-minute training session on ultrasound-guided pericardiocentesis held on a single day.

We used two different training models for ultrasoundguided pericardiocentesis, as described below:

\section{Agar-based model}

The first model was an agar-based model with a waterfilled balloon embedded within, to simulate the pericardial sac, as previously described by Zerth et al. ${ }^{16}$ Ultrasound-compatible agar jelly was used to represent subcutaneous tissues. This model permitted aspiration of fluid within the balloon, but not insertion of a drain due to the friable nature of the agar jelly to the larger-bore drain. For this training session that included 20 participants, three sets of the agar-based model were prepared.

\section{Psyllium-based model (Centesys)}

A new model was designed using psyllium husk to simulate subcutaneous tissue. Psyllium husk has been used previously for other ultrasound-compatible simulation models to simulate soft tissue. ${ }^{18,19}$ Part of a rubber ball was stretched over a plastic container to mimic the pericardium and replicate a "give" during needle puncture. A ping-pong ball was placed within a plastic container to resemble the heart. An electric pump (Mingy ornament water pump [Guangdong, China], model MY-18, power 2.5 watts, maximum output 180 litres per hour) was used to pump water continuously into the container to maintain the shape and tension of the rubber ball (Fig. 1).

The container was placed on an acrylic stand to angle it such that the subxiphoid ultrasound window could be simulated. This set-up was then put into a larger container filled with water, and the psyllium and a skin layer made of silicone (Dragon Skin ${ }^{\mathrm{TM}}$ FAST [Pennsylvania, US])

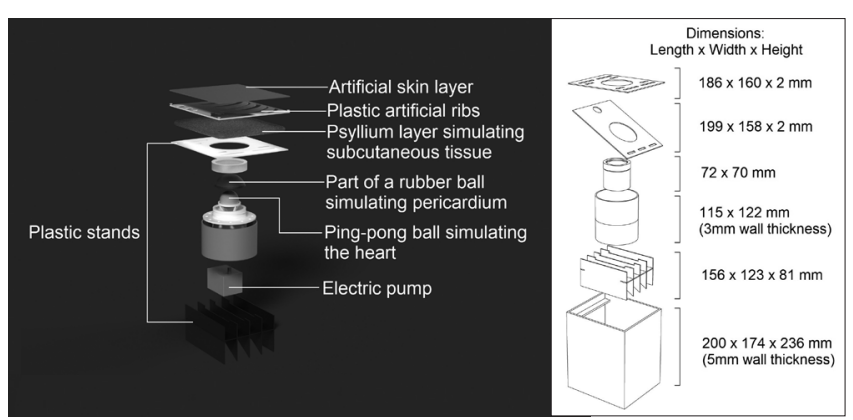

Fig. 1. Diagram showing internal layout of Centesys (left) and dimensions of different components of Centesys (right). 
were placed on top of it. The dimensions of the set-up can be found in Fig. 1. This newer model was ultrasoundcompatible (Fig. 2), and enabled needle aspiration of fluid as well as insertion of a pericardial drain (Fig. 3).

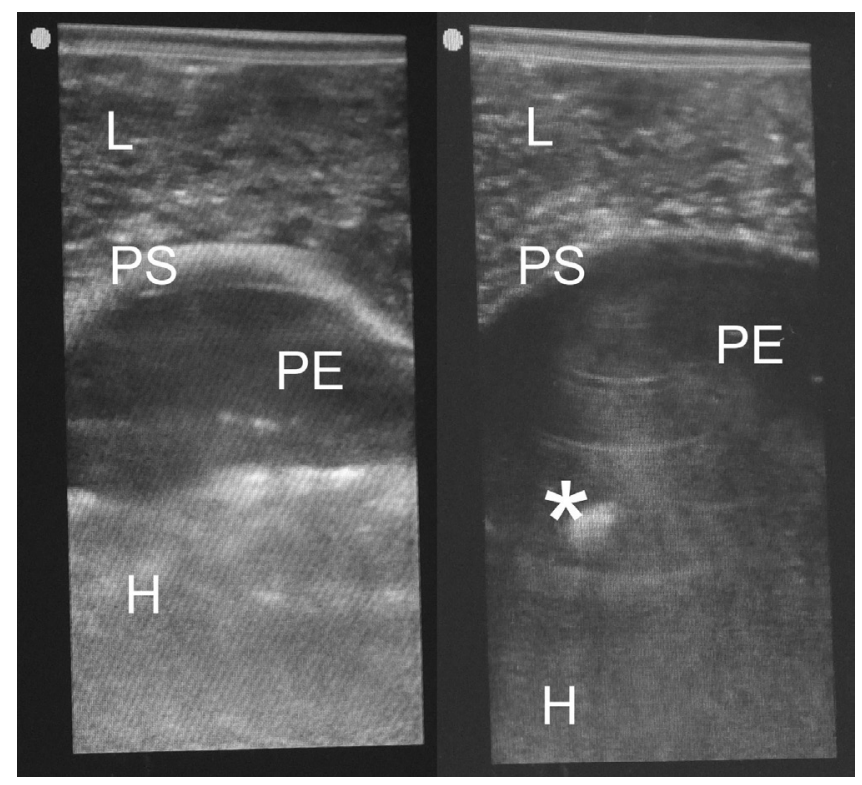

Fig. 2. Ultrasound images from Centesys showing: (left) subxiphoid window showing liver (L), pericardial sac (PS), pericardial effusion (PE), and heart $(\mathrm{H})$; (right) subxiphoid window showing similar anatomical structures with needle in-situ (shown by*) within pericardial effusion.
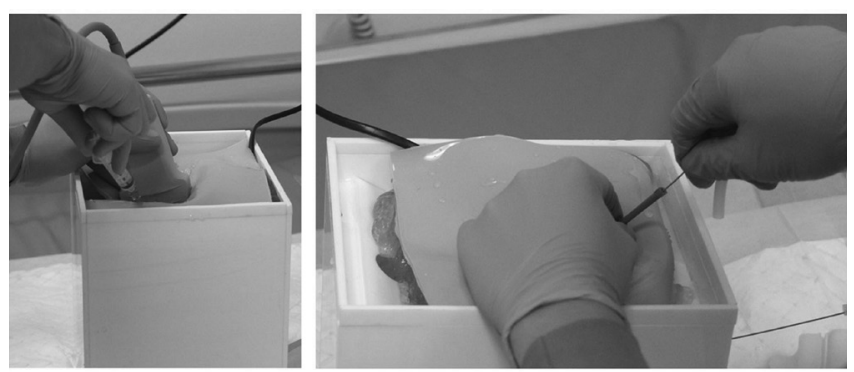

Fig. 3. Photographs of Centesys, showing (left) ultrasound-guided needle aspiration, (right) pericardial drain insertion.

For the training session, one set of the Centesys model was utilised.

Each participant was given a total of 45 minutes to complete the pericardiocentesis training with both models (20 minutes for training with each model, with a 5-minute buffer time in between). Participants were tasked to aspirate fluid from the simulated pericardial sac using a needle for both models, followed by insertion of a pericardial drain (only for Centesys). Two trained assessors observed the participants for their procedural skills and evaluated their performances in the form of an objective structured clinical examination (OSCE) with both models using a competency checklist adapted from a standardised source ${ }^{20}$ as part of an educational exercise. The OSCE results were formative and are not presented as part of the study. The assessors independently evaluated each participant and there was no discussion between the assessors during completion of the checklist.

Participants were asked to rate their confidence in performing various steps of ultrasound-guided pericardiocentesis (e.g. obtaining a subxiphoid view for cardiac ultrasound, assessing presence of pericardial effusion on cardiac ultrasound, and overall confidence in performing the procedure) on a 7-point Likert scale (with 1 being little to no confidence or knowledge, and 7 being extremely confident or competent, respectively) before and after the training session with each model. They were also asked to rate the quality of the visualised structures on ultrasound with both models from a Likert scale of 1 to 7 ( 1 being not clear and 7 being extremely clear); realism of the visualised structures in comparison to cardiac anatomy seen on ultrasound, and preference for the two models on a 7-point Likert scale immediately after training with each model. The effectiveness of the models was determined based on whether participants were able to complete the task (e.g. aspiration of pericardial fluid and insertion of a pericardial drain for Centesys, and aspiration of pericardial fluid for the agar-based model) by the end of the training session.

Web-based randomisation method ${ }^{21}$ was used to determine if each participant would undergo pericardiocentesis training using the agar-based model or Centesys first. Blinding was not possible given the nature of the interventions.

\section{Statistical analysis}

Categorical variables are reported in proportions while continuous variables are reported in median with corresponding interquartile range (IQR) as appropriate. All data were populated in Microsoft Excel (Microsoft Corp, Redmond, US). Upon completion of data collection electronically, all data were verified by two investigators independently. Differences in categorical variables for unmatched groups were compared with chisquare test or Fisher's exact test, as appropriate. Skewed continuous variables for matched groups were analysed using Wilcoxon signed-rank test. Statistical significance was set at $P<0.05$. 
To avoid carryover effects, only responses with the first assigned model were analysed for questions regarding confidence in carrying out the procedure. For other questions pertaining to the fidelity of and satisfaction with the models, all responses were considered.

\section{Results}

A total of 20 participants were recruited, comprising 12 junior residents, 6 senior residents and 2 resident physicians. The overall median postgraduate year was 4 (interquartile range [IQR] 3.75-6) years. There was no significant difference in postgraduate years of the participants in the two groups (median 4 years, IQR 4-5.75 years for the group starting with the psyllium-based model versus median 5 years, IQR 3-6 years for the agar-based model, $P=0.684$ ). For the 18 residents, there was a median of 36 hours (IQR 12-42 hours) of dedicated ultrasound scanning (conducted as part of residency training), with no significant difference between the intervention and control groups (median 42 hours, IQR 24-42 hours for the group starting with the psyllium-based model and median 24 hours, IQR 12-42 hours for the agar-based group, $P=0.730$ ). None of the participants had prior real-life experience in performing pericardiocentesis.

\section{Confidence in various steps of pericardiocentesis}

Prior to commencing the training session, participants in the Centesys group rated themselves a median score of 2.5 (IQR 2-4) for their level of confidence in performing pericardiocentesis, which improved to 5 (IQR 4-6) after training with Centesys $(P=0.007)$ (Table 1$)$. In the agar-based model, participants' self-rated confidence increased from a median score of 2 (IQR 1-4) to 3.5 (IQR 3-5) after training $(P=0.036)$. The differences between the pre-test confidence scores between both groups were not significant $(P=0.434)$.
Likewise, there was improvement in confidence of participants for obtaining a subxiphoid ultrasound view on cardiac ultrasound in the Centesys group from a median score of 4.5 (IQR 3.75-5) pre-training to 5 (IQR 4.75-5.25) post-training $(P=0.034)$. However, there was no significant difference between pre- and post-training scores for participants in the agar-based model group. The pre-test confidence scores for obtaining a subxiphoid ultrasound view $(P=0.670)$ were not significantly different between both the agar-based model and Centesys groups.

\section{Ultrasound image quality}

Participants consistently rated Centesys as having superior quality of visualised cardiac anatomical structures-such as the heart, pericardium, and pericardial effusion-compared to the agar-based model (Table 2). Participants also reported improved visualisation of the needle when using Centesys (median 5 [IQR 5-6]) compared to the agar-based model (median 4.5 [IQR 4-5]) $(P=0.013)$. Centesys was regarded to be more realistic in simulating cardiac anatomy on ultrasound (median 5 [IQR 4-5]) in contrast to the agar-based model (median 3.5 [IQR 3-4]) $(P=0.002)$.

\section{Effectiveness and learners' satisfaction of model as a training tool}

By the end of the training session with Centesys, all 20 participants were able to insert the pericardial drain into the simulated pericardial effusion and aspirate fluid from the Centesys model. For the agar-based model, all 20 participants were also able to aspirate pericardial fluid from the model; however, this model did not allow for pericardial drain insertion. There was also greater satisfaction reported with Centesys (median 5 [IQR 5-6] versus 4 [IQR 3.75-4], $P<0.001)$.

Table 1. Participants' self-assessment of confidence of ultrasound-guided pericardiocentesis after training with initial model*

\begin{tabular}{|c|c|c|c|c|c|c|}
\hline \multirow{2}{*}{ Variables } & \multicolumn{3}{|c|}{ Agar-based model $(n=10)$} & \multicolumn{3}{|c|}{ Centesys $(n=10)$} \\
\hline & Pre-training & Post-training & $P$ value & Pre-training & Post-training & $P$ value \\
\hline $\begin{array}{l}\text { Confidence in obtaining subxiphoid view for cardiac } \\
\text { ultrasound }\end{array}$ & $4(3-6)$ & $4(3-5.25)$ & 0.577 & $4.5(3.75-5)$ & $5(4.75-5.25)$ & 0.034 \\
\hline $\begin{array}{l}\text { Confidence in assessing for pericardial effusion on } \\
\text { cardiac ultrasound }\end{array}$ & $4(3-5.25)$ & $4(3.75-5.25)$ & 0.317 & $5(4-5)$ & $5(4.75-6)$ & 0.102 \\
\hline Confidence in doing pericardiocentesis & $2(1-4)$ & $3.5(3-5)$ & 0.036 & $2.5(2-4)$ & $5(4-6)$ & 0.007 \\
\hline
\end{tabular}

*Data presented in median (interquartile range) unless otherwise specified 
Table 2. Participants' perception of ultrasound image quality in the pericardiocentesis models*

\begin{tabular}{|c|c|c|c|}
\hline Variables & $\begin{array}{l}\text { Agar-based model } \\
\qquad(\mathrm{n}=20)\end{array}$ & $\begin{array}{l}\text { Centesys } \\
(n=20)\end{array}$ & $P$ value \\
\hline How clearly can the heart be visualised in the model? & $4(3-4)$ & $5(4-6)$ & 0.005 \\
\hline How clearly can the pericardium be visualised in the model? & $4(3-4)$ & $5(4-6)$ & 0.013 \\
\hline How clearly can the pericardial effusion be visualised in the model? & $4(3-5)$ & $5.5(5-6)$ & 0.002 \\
\hline How clearly is the needle tip visible? & $4.5(4-5)$ & $5(5-6)$ & 0.013 \\
\hline How realistic is the model in simulating cardiac anatomy on ultrasound? & $3.5(3-4)$ & $5(4-5)$ & 0.002 \\
\hline
\end{tabular}

*Data presented in median (interquartile range) unless otherwise specified

\section{Discussion}

Pericardiocentesis is a high-risk but critical procedure that occurs rarely in the emergency department, making simulation indispensable to maintain competency. In our study, participants expressed their increased confidence in performing the procedure after undergoing simulation training; to a greater degree with the novel psylliumbased model compared to the agar model.

Furthermore, while there was noted to be an overlap in the IQRs for all questions, the psyllium-based model consistently achieved statistically significantly higher median scores for realism and clarity of images. After numerous trials of material selection, psyllium was chosen as it closely mimics the characteristics of subcutaneous tissue in terms of density, self-healing properties and ultrasound echogenicity.

The inferior quality of ultrasound images in the agar-based model was likely due to increased irreversible artefacts and distortion of the ultrasound images with every attempt from additional burrows through the agar caused by needles. This loss of fidelity was also contributed by deflation of the balloon after an average of 5 puncture attempts had been made. We attempted to circumvent this issue and maintain consistency in the image quality for each user for the agar-based model by preparing three models for the session, such that a fresh model could be used once the image quality was deemed to be too poor. However, there was still a certain degree of loss of fidelity after each use, which was unavoidable as it was not practical to fabricate a model for every single participant.

In addition, the balloon in the agar-based model may not have had adequate elasticity to produce a realistic "give", which was appreciable in Centesys as the tension of the pericardial sac (i.e. part of the stretched rubber ball) was maintained through the use of an electric pump that generated consistent hydrostatic pressure (Fig. 1).
Participants were more satisfied with the psylliumbased model as a training tool compared to the agarbased model. We postulate that this was because of the surprising durability of psyllium, which accommodated the insertion of a larger bore and bulkier pericardial drain, thus making it more realistic and enabled simulation of the entire pericardiocentesis procedure.

In our institution, simulation sessions for ultrasoundguided pericardiocentesis are held yearly. This session is open to both specialists and residents to maintain competency as few have had the chance to perform the procedure in real life. Each session is attended by about 20 physicians. The agar-based model is labour intensive as 3 to 4 models are required per training session to compensate for the loss of fidelity with each repeated attempt as described above. Each model takes approximately 1 hour to complete from preparation of materials to assemblage of the model, and an additional 2 hours are required for the agar to harden to a firm consistency while refrigerated.

With the novel model, only the psyllium component had to be replaced every session, as it tended to disintegrate after one week. However, this step is inexpensive as a $150 \mathrm{~g}$ packet of psyllium costs approximately US\$5.50, and each sheet (enough for one simulation session) consumes only 4 tablespoons worth of psyllium (approximately $24 \mathrm{~g}$ ). The entire process of preparing the psyllium takes approximately 15 minutes and involves boiling the psyllium and stirring the solution at 5-minute intervals until a jelly-like consistency is obtained. The other component requiring replacement is the rubber ball that is used to represent the pericardial sac. The rubber material is self-sealing and able to withstand more than 200 punctures without losing its elasticity when tested at a separate setting. The ball is also a low-cost component value of approximately US $\$ 1.70$ and could be replaced in the model easily by sliding it under the screwed-on container lid. 
The other parts of the model which do not need replacement are also relatively inexpensive, such as the electric pump, which was purchased at a local store for approximately US $\$ 3.70$, but the same model can be purchased online from US\$5.00-\$9.00..$^{22,23}$ If required, a less expensive model with similar specifications can also be sourced locally. The silicone skin was made from Dragon Skin ${ }^{\mathrm{TM}}$ FAST silicone, and a $400 \mathrm{ml}$ quart can be purchased online at US $\$ 25.36 .{ }^{24}$ This component also does not require replacement with every use. Table 3 details the list of materials and estimated cost price of Centesys and the agar-based model.

\section{Limitations}

The novel psyllium-based model had some limitations. It lacked anatomical landmarks that would have been useful in teaching the insertion point for pericardiocentesis. Further refinements, such as incorporating an overlying phantom to guide surface anatomical landmarks, can be made. This new model also requires the use of an electric pump to continuously generate water pressure from the environment into the container to maintain tension of the rubber ball, thereby necessitating a power outlet for it to work and thus rendering it less portable.

After training with the first model, the participants may have had increased confidence in performing pericardiocentesis from the first round of training and hence may have given higher confidence scores to the second model (i.e. carryover effects). To overcome this potential bias, we only considered the results from training with the first model that the participants were randomised to for questions pertaining to confidence. This resulted in a reduced sample size in terms of power analysis.

Blinding was not possible due to the nature of the study as the learners were required to interact with the models. Since the outcomes were subjective in nature, this may have introduced bias into the learners' ratings.

For the OSCE session, no objective evaluation was carried out to assess inter-rater reliability. However, the assessors were given a briefing prior to the session in order to standardise how the station should be carried out (e.g. how many prompts were allowed, how the checklist should be marked, etc.). The checklist was also objective and only gave marks if certain actions were fulfilled by the participant. Moreover, the OSCE results were formative and are not presented as part of the study.

The models were tested on a relatively small number of participants from a single centre who had no prior experience with pericardiocentesis. Therefore, the results may not be generalisable to advanced learners from other clinical specialties such as cardiologists, cardiothoracic surgeons or intensivists who may be more familiar with the procedure. We are also unable to establish if the improvement in simulation performance and self-rated scores will translate to higher proficiency

Table 3. Materials used and cost price for assembly of pericardiocentesis models

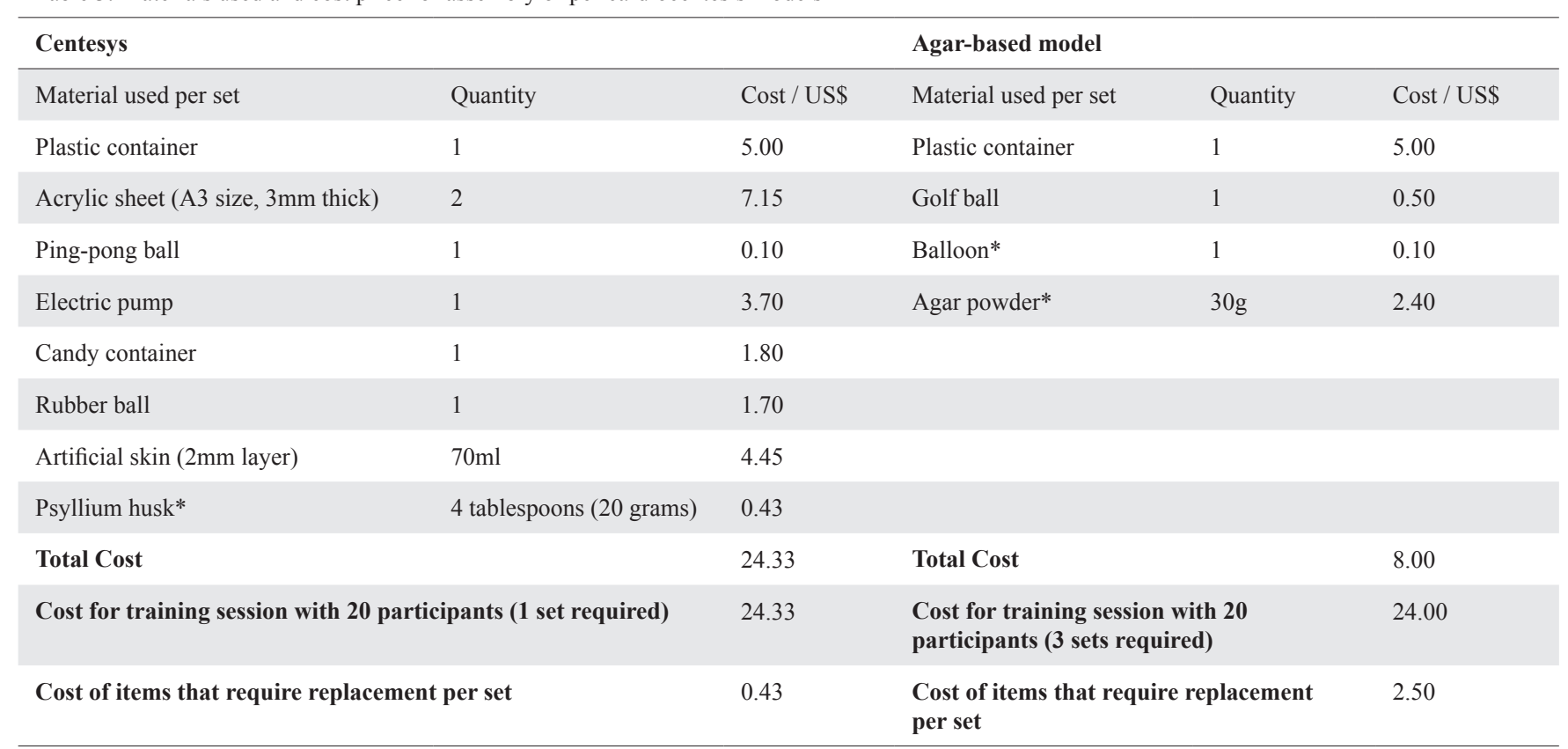

*Denotes component that requires replacement after each training session 
in performing pericardiocentesis in real life. However, as emergency medicine residents in our institution undergo a structured bedside ultrasound programme and are familiar with ultrasound images of cardiac anatomy, they are able to gauge the realism and quality of the ultrasound images provided by both models.

\section{Conclusion}

The new psyllium-based model was perceived to be more realistic in simulating cardiac anatomy on ultrasound compared to the agar-based model, had greater learner satisfaction, and was an effective tool for teaching ultrasound guided pericardiocentesis and drain insertion. Further enhancements to the novel model are required to improve the overall realism and portability.

\section{REFERENCES}

1. Spodick DH. Acute cardiac tamponade. N Engl J Med 2003; 349:684-90

2. Kalivoda EJ, Sullivan A, Bunting L. A cost-effective, rapidly constructed simulation model for ultrasound-guided pericardiocentesis procedural training. J Emerg Med 2019;56:74-9.

3. Willner DA, Grossman SA. Pericardiocentesis. [Updated 30 Aug 2020]. In: StatPearls. Treasure Island (US): StatPearls Publishing; Jan 2020. Available at: https://www.ncbi.nlm.nih.gov/books/ NBK470347/. Accessed on 20 October 2020.

4. Maggiolini S, Gentile G, Farina A, et al. Safety, efficacy, and complications of pericardiocentesis by real-time echo-monitored procedure. Am J Cardiol 2016;117:1369-74.

5. Tsang TSM, Enriquez-Sarano M, Freeman WK, et al. Consecutive 1127 therapeutic echocardiographically guided pericardiocenteses: clinical profile, practice patterns, and outcomes spanning 21 years. Mayo Clin Proc 2002;77:429-36.

6. Cho BC, Kang SM, Kim DH, et al. Clinical and echocardiographic characteristics of pericardial effusion in patients who underwent echocardiography guided pericardiocentesis: Yonsei Cardiovascular Center experience, 1993-2003. Yonsei Med J 2004;45:462-8.

7. Buchanan CL, Sullivan VV, Lampman R, et al. Pericardiocentesis with extended catheter drainage: an effective therapy. Ann Thorac Surg 2003;76:817-20.

8. Schube J, Toro DF. 2017 Innovations. Acad Emerg Med 2017;24: S282-98.
9. Chua MT, Chan GWH, Kuan WS. Reversible causes in cardiovascular collapse at the emergency department using ultrasonography (REVIVE-US). Ann Acad Med Singap 2017;46:310-6.

10. GT Simulators. Ultrasound-Guided Pericardiocentesis Simulator. Available at: https://www.gtsimulators.com/ultrasound-guidedpericardiocentesis-simulator-p/kk-mw15.htm. Accessed on 20 October 2020

11. GT Simulators. Transthoracic Echocardiography And Pericardiocentesis Ultrasound Training Model. Available at: https://www.gtsimulators.com/transthoracic-echocardiographypericardiocentesis-p/bp-tte1701.htm. Accessed on 20 October 2020.

12. Daly R, Planas JH, Edens MA. Adapting gel wax into an ultrasoundguided pericardiocentesis model at low cost. West J Emerg Med 2017;18:114-6.

13. Campo dell'Orto M, Hempel D, Starzetz A, et al. Assessment of a low-cost ultrasound pericardiocentesis model. Emerg Med Int 2013;2013:1-7.

14. Inboriboon PC, Lumlertgul S. A cadaveric model for pericardiocentesis training. J Emerg Med 2013;44:661-2.

15. Habicht JL, Kiessling C, Winkelmann A. Bodies for anatomy education in medical schools: an overview of the sources of cadavers worldwide. Acad Med 2018;93:1293-300.

16. Zerth H, Harwood R, Tommaso L, et al. An inexpensive, easily constructed, reusable task trainer for simulating ultrasound-guided pericardiocentesis. J Emerg Med 2012;43:1066-9.

17. Peng LL, Ooi SBS. Emergency medicine residency programme in Singapore-where are we at since inception? Ann Acad Med Singap 2015;44:77-8.

18. Kwon SY, Hong SH, Kim ES, et al. The efficacy of lumbosacral spine phantom to improve resident proficiency in performing ultrasound-guided spinal procedure. Pain Med 2015;16:2284-91.

19. Sullivan A, Khait L, Favot M. A novel low-cost ultrasound-guided pericardiocentesis simulation model: demonstration of feasibility. J Ultrasound Med 2018;37:493-500.

20. Trivedy C, Hall M, Parfitt A. Mastering emergency medicine: A practical guide. 1st edition. Boca Raton: CRC Press; 2009, p437.

21. Dallal GE. Randomization.com. Available at: http://randomization. com/. Accessed on 20 October 2020.

22. YOYCART.com. My-018 aquarium mini fish tank water pump. Available at: https://www.yoycart.com/Product/37226783097. Accessed on 20 October 2020.

23. Aliexpress.com. MY-018 Mini submersible pump water pump safety static process $2.5 \mathrm{~W}$ lift $0.55 \mathrm{M}$. Available from: https://www.aliexpress. com/item/32839341187.html. Accessed on 20 October 2020 .

24. Smooth-on Store. Dragon skin ${ }^{\mathrm{TM}} 10$ FAST. Available at: https://shop. smooth-on.com/dragon-skin-10-fast. Accessed on 20 October 2020. 